\title{
Jérôme Lejeune 1927-1994
}

\author{
Jean de Grouchy
}

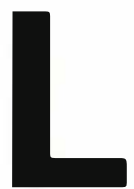

e Professeur Jérôme Lejeune est décédé, à 67 ans, emporté par un cancer. Un mal à l'étude duquel il avait consacré une partie de son activité de recherche.

J. Lejeune était un enfant de Montrouge. Ainsi qu'il devait le dire au cours de sa leçon inaugurale à la Faculté de Paris, il avait choisi ses stages hospitaliers en fonction de la proximité d'une gare commode. Nous avons fait connaissance à la caserne des Batignolles, en route pour notre service militaire en Allemagne. Lui venait d'entrer "en génétique " dans l'équipe du Professeur Raymond Turpin et moi-même dans celle du Professeur Maurice Lamy. Malgré cette rivalité au sommet, nous sommes demeurés, jusqu'à sa fin, les meilleurs amis du monde.

Très tôt, J. Lejeune fut fasciné par l'énigme que posait le mongolisme. Toutes les hypothèses avaient été émises pour expliquer l'étiologie de cette "condition": hérédité récessive, hérédité dominante, anomalie chromosomique. J. Lejeune pensait qu'il pouvait s'agir d'une anomalie semblable à la duplication Bar décrite chez la drosophile. Il rencontra dans le service le Dr. Marthe Gautier qui lui apprit les techniques de culture de fibroblastes qu'elle venait d'acquérir aux Etats-Unis. Cette collaboration, ainsi que la mise au point des "premières" techniques de cytogénétique, abou-
Comptes Rendus de l'Académie des Sciences d'une note décrivant la présence chez les enfants mongoliens d'un petit chromosome acrocentrique surnuméraire. Cette découverte, de ce qui devint plus tard la trisomie 2l, revêt une importance considérable puisqu'elle ouvrait la voie à une science nouvelle, la cytogénétique, humaine d'abord, animale ensuite. Son développement, exponentiel en de multiples directions, devait permettre de connaître les secrets du matériel héréditaire.

Les découvertes de J. Lejeune en cytogénétique s'enchaînèrent rapidement : la description de la maladie du cri-du-chat, par délétion de $5 p$; la première translocation entre grands acrocentriques; de nombreuses aneusomies partielles; la théorie des anneaux... Il proposa le concept de type et contretype, selon lequel deux syndromes dus, l'un à une monosomie, et l'autre à une trisomie, pour le même segment chromosomique, s'opposent par leurs signes cliniques, par exemple brachy- versus dolichomésophalangie.

Autre contribution, la gémellité monozygote hétérocaryote, caractérisée par l'existence de jumeaux “identiques» par leur groupes sanguins et leur tolérance immunologique à la greffe de peau réciproque, mais qui diffèrent par une seule paire de chromosomes. La première observation de J. Lejeune concernait des jumeaux, l'un mâle, 46, XY et l'autre atteint d'un syndrome de
Turner, 45, X. Ils résultaient, selon lui, de deux accidents survenus simultanément, à savoir la formation de jumeaux monozygotes et la formation d'une mosaique $46, \mathrm{XY} / 45$, $X$ par perte de l'Y chez l'un des jumeaux. D'autres observations ont été publiées par J. Lejeune, en particulier un couple monozygote trisomique 21 /normal.

Et surtout! J. Lejeune a eu recours à cette forme de gémellité pour expliquer la survenue, au cours de l'évolution des espèces, d'un couple ancestral mâle/femelle dont les individus étaient porteurs d'un même remaniement, la translocation du chromosome 2, réduisant le nombre chromosomique de $\mathrm{N}=48$ chez les Pongidés à $\mathrm{N}=46$ chez l'homme. Ce couple pouvait n'être autre qu'Adam et Eve.

Cytogénétique clinique, évolutive, gémellaire, J. Lejeune a également contribué à la connaissance de la cytogénétique cancéreuse en rapportant la première observation d'une évolution clonale chez un enfant trisomique leucémique. Avec tout le regret qu'on imagine, il s'est également rendu compte a posteriori qu'il avait, sur une de ses lames de leucémie myéloïde chronique, un superbe chromosome de Philadelphia "princeps" qui lui avait... échappé. Même, dans les dernières semaines de sa vie, connaissant son mal, il s'était à nouveau tourné vers la recherche d'une explication de la carcinogenèse. Avec son médecin traitant, le Prof esseur Lucien Israël, ils cherchèrent à corréler la cancé- 
rogenèse et l'évolution du système nerveux chez les organismes supérieurs. Quelques jours à peine avant sa fin, Jérôme m'avait téléphoné pour me dire qu'il était frappé par le fait que le cancer n'apparaît qu'après un certain degré d'évolution phylogénétique des êtres vivants, épargnant les espèces les plus primitives. Il me demanda si j'avais la moindre notion à cet égard. Je n'ai pu lui répondre que " hélas non".

Mais là ne s'arrête pas l'activité créatrice de J. Lejeune. Il se passionna durant un temps pour la biochimie stérique. Il avait fait construire "à façon" des modèles moléculaires, ses "boules", qui envahirent comme des coquelicots les meubles de son laboratoire de la rue des Saints-Pères. Ses thèmes de recherche pouvaient être aussi divers que la transmission du courant nerveux dans les synapses ou la mise en évidence d'un " code de congruence " expliquant la reconnaissance des séquences d'ADN par les molécules protéiques lors des régulations génétiques ou hormonales.
Mais l'obsession de J. Lejeune a toujours été de déceler les mécanismes de la débilité mentale afin de la traiter. Et ce fut, tout au long des dernières années, le tourbillon des flux biochimiques s'entraînant les uns les autres à la manière d'engrenages circulaires. Ses dessins à la plume laissaient bouche bée. Son cheval de bataille était la famille des folates dont le rôle était à ses yeux fondamental.

L'expansion imaginative de Jérôme Lejeune était quasi infinie, produisant chaque jour une nouvelle idée, un nouveau concept, une nouvelle théorie. Juste pour l'anecdote, il avait noté qu'il n'existe pas dans la Nature de surface plane... sauf le plan de l'iris de l'œil des animaux. Etonnante observation!

Professeur de Génétique Fondamentale, J. Lejeune était couvert d'honneurs: membre de l'Institut, membre de l'Académie des Sciences Pontificale, membre de l'Académie de Médecine, docteur honoris causa de nombreuses universités étrangères. Il ne fut jamais lauréat Nobel, alors que son œuvre scientifique l'aurait pleinement justifié. Et cela pour des raisons qui échappent aux considérations purement scientifiques. Elles sont nombreuses. Elles font partie de "l'environnement" extrascientifique du "savant" Lejeune. Nous savons tous que ses convictions morales, proclamées urbi et orbi, ont suscité vénération et opprobre.

Je veux dire très simplement, pour terminer, que lors de ses obsèques, la cathédrale Notre Dame de Paris était comble, remplie de personnes venues du monde entier, témoignant de la notoriété et de l'estime universelles de Jérôme Lejeune. Je veux dire aussi qu'il a laissé une épouse exemplaire qui a été sa compagne indéracinable depuis les premiers jours, cing enfants, et vingt petits enfants. Un témoignage irréfutable de son respect de la vie

\section{J. de Grouchy}

Directeur de Recherche honoraire au CNRS, la Ferme aux Jasmins, 45, route de Saint Franfois, 06130 Grasse, France. 